\title{
ORIGINAL
}

\section{Evaluation of Plasma, Salivary, and Urinary Cortisol Levels for Diagnosis of Cushing's Syndrome}

\author{
SATORU SAKIHARA ${ }^{1)}$, KAZUNORI KAGEYAMA ${ }^{1)}$, YUtAKA OKI ${ }^{2)}$, MASARU DOI ${ }^{3)}$, YASUMASA IWASAKI ${ }^{4)}$, \\ SHINOBU TAKAYASU ${ }^{1)}$, TAKAKO MORIYAMA ${ }^{1)}$, KEN TERUI ${ }^{1)}$, TAKESHI NIGAWARA ${ }^{1)}$, YUKIO HIRATA ${ }^{3)}$, \\ KOZO HASHIMOTO ${ }^{4)}$ AND TOSHIHIRO SUDA ${ }^{1)}$ \\ 1) Department of Endocrinology and Metabolism, Hirosaki University Graduate School of Medicine, Aomori 036-8562, Japan \\ 2) Second Division, Department of Medicine, Hamamatsu University School of Medicine, Shizuoka 431-3192, Japan \\ 3) Department of Clinical and Molecular Endocrinology, Tokyo Medical and Dental University Graduate School, Tokyo 113-8510, Japan \\ 4) Department of Endocrinology, Metabolism and Nephrology, Kochi Medical School, Kochi 783-8505, Japan
}

\begin{abstract}
As a screening test for Cushing's syndrome, the evaluation of late-night cortisol levels is indispensable. We evaluated the usefulness and accuracy of plasma, urinary, and salivary cortisol levels measured late at night for the diagnosis of Cushing's syndrome. High cortisol levels ( $>5 \mu \mathrm{g} / \mathrm{dL}$ ) during the night are indicative of Cushing's syndrome, although night plasma cortisol levels are not readily reproducible because of the stressful situation. There was no correlation between plasma and urinary cortisol levels late at night, and late-night urinary cortisol levels provided weak information for the diagnosis of Cushing's syndrome. By contrast, late-night plasma and salivary cortisol levels showed a positive correlation, and salivary cortisol sampling was found to be useful for the diagnosis of Cushing's syndrome, because more than $0.4 \mu \mathrm{g} / \mathrm{dL}$ of late-night salivary cortisol levels gave a sensitivity of $86 \%$ and a specificity of $100 \%$ in our hospital. This method is also useful for the diagnosis of early or mild stage Cushing's syndrome, so-called subclinical Cushing's syndrome. Inherent differences between assays make it difficult to define optimal diagnostic criteria. However, the relative levels of salivary cortisol ratio, which is presented as a relative level, compared with the mean levels of healthy subjects in each institute, is useful for the screening of Cushing's syndrome as the cut-off level of 1.5 shows both high sensitivity and specificity in subclinical and overt Cushing's syndrome. Late-night salivary cortisol measurement is therefore a primary method of choice in the screening of patients suspected of having Cushing's syndrome.
\end{abstract}

Key words: ACTH, Adrenal gland, Cortisol, Cushing's disease, Pituitary

CUSHING'S SYNDROME results from a chronic glucocorticoid excess usually caused by cortisol- or adrenocorticotropic hormone (ACTH)-secreting tumors [1]. Sufferers of the disorder have a Cushingoid appearance, with clinically characteristic signs and symptoms including central obesity, moon face, skin atrophy, purple striae and buffalo hump [2]. An absence of typically clinical features in Cushing's syndrome has been defined as subclinical Cushing's syndrome [3].

The dexamethasone suppression test (DST) and

Received Nov. 25, 2009; Accepted Jan. 12, 2010 as K09E-340 Released online in J-STAGE as advance publication Feb. 7, 2010

Correspondence to: Kazunori KAGEYAMA, M.D., Department of Endocrinology and Metabolism, Hirosaki University Graduate School of Medicine, 5 Zaifu-cho, Hirosaki, Aomori 036-8562, Japan. E-mail:kkageyama@hkg.odn.ne.jp corticotropin-releasing hormone (CRH) test are well defined for the diagnosis of Cushing's syndrome [4-6], while desmopressin has been used for the differential diagnosis of ACTH-dependent Cushing's syndrome [7, 8]. However, it is clear that the diagnosis and differential diagnosis of Cushing's syndrome are still challenging problems in clinical endocrinology $[9,10]$.

Cortisol is an important steroid in the regulation of stress response [11]. It exists in both free (active) and protein-bound forms in the blood, but only in a free form in saliva [11]. Evans et al. first studied the feasibility of late-night salivary cortisol measurements for the clinical evaluation of endogenous hypercortisolism in the 1980s [12]. Since then, these measurements have been shown to offer high sensitivity and specificity in the diagnosis of Cushing's syndrome [13].

The diagnostic criteria for Cushing's syndrome 
were reported as Endocrine Society Clinical Practice Guidelines in 2008 [14] and include the following endocrinological findings as initial tests: 1) an upper limit of normal levels of urine free cortisol, 2) high late-night salivary cortisol levels $(>0.14 \mu \mathrm{g} / \mathrm{dL}), 3)$ incomplete suppression of cortisol ( $>5 \mu \mathrm{g} / \mathrm{dL}$ ) by a low-dose (1 mg) overnight DST, and 4) incomplete suppression of cortisol ( $>1.4$ or $1.8 \mu \mathrm{g} / \mathrm{dL}$ ) by a longer low-dose $(2 \mathrm{mg} /$ day for $48 \mathrm{~h})$ DST. A low-dose DST and CRH test, and midnight serum cortisol test (sleeping, $>1.8 \mu \mathrm{g} / \mathrm{dL}$; awake, $>7.5 \mu \mathrm{g} / \mathrm{dL}$ ) are also considered high sensitivity tests.

The first diagnostic criteria for Cushing's disease were reported by the working group of the Ministry of Health, Labour, and Welfare of Japan in 2003, and screening tests were renewed in 2007 [4, 15]. In discussing the present diagnostic criteria, we refer to our extensive data on plasma, salivary, and urinary cortisol levels. Therefore, in the present study, we evaluated the usefulness and accuracy of plasma, salivary and urinary cortisol levels for the diagnosis of Cushing's syndrome.

\section{Materials and Methods}

\section{Study 1}

Nineteen subjects with normal ACTH and cortisol secretory dynamics from Hirosaki University School of Medicine \& Hospital, Japan were enrolled in the study to determine the reproducibility of measuring plasma cortisol levels during night time sleep. Blood collection at 23:00 was performed twice, first within three days after study entry and, second, over the following week.

Fifty subjects at Hirosaki University School of Medicine \& Hospital were enrolled in the study for Pearson's correlation analysis between plasma and salivary cortisol levels. A total of 72 blood and salivary samples were taken at 23:00 and/or 6:00 under fasting condition. The DST, human CRH test, and desmopressin test were performed to evaluate ACTH and cortisol secretory dynamics. Five subjects were diagnosed with Cushing's disease, and one subject was diagnosed with subclinical Cushing's disease, due to the presence of pituitary adenomas. Two cases were diagnosed with adrenal Cushing's syndrome, and six with subclinical adrenal Cushing's syndrome. These diagnoses were confirmed after the operations. Thirtysix subjects, which showed normal ACTH and cortisol secretory dynamics by such screening tests, were used as controls. Blood and salivary samples taken at 23:00 were used to obtain a scatterplot of plasma and salivary cortisol levels. Patient informed consent was obtained before commencing the study, and all tests were performed according to clinical guidelines.

\section{Study 2}

A total of 114 subjects from Hirosaki University School of Medicine \& Hospital $(n=35)$, Hamamatsu University School of Medicine \& Hospital, Japan (n =35), Tokyo Medical and Dental University Graduate School \& Hospital, Japan $(\mathrm{n}=27)$ and Kochi Medical School \& Hospital, Japan ( $n=17)$ were enrolled in the study to analyze salivary cortisol ratios. Of these, 27 subjects were diagnosed with Cushing's syndrome and 10 subjects with subclinical Cushing's syndrome; 77 subjects with normal ACTH and cortisol secretory dynamics were used as controls. The same 114 subjects were enrolled in the study for Pearson's correlation analysis between plasma and salivary cortisol levels.

Blood and salivary samples in a recumbent position, then urinary samples were taken at 23:00. Data on urinary cortisol levels were obtained from a total of 67 subjects. Salivary cortisol ratios are presented as a relative level, compared with the mean levels of healthy subjects from each institute.

\section{Assays}

Plasma and urinary cortisol levels were measured by radioimmunoassay (RIA) (TFB Co., Tokyo, Japan, or Incstar Co., Stillwater, MN, USA). Salivary cortisol levels were measured by electrochemiluminescence-immunoassay (ECLIA) using an assay kit (Roche Diagnostics, Mannheim, Germany) in Hirosaki University Graduate School of Medicine and Kochi Medical School. RIA (Incstar Co.) was perfomed in Tokyo Medical and Dental University Graduate School, and enzyme immunoassay (Salimetrics Inc., State College, PA, USA) was carried out in Hamamatsu University School of Medicine.

\section{Statistical Analysis}

Correlation analysis between plasma and salivary cortisol levels or ratios was performed by Pearson's correlation analysis. Statistical analyses of data were performed using Kruskal-Wallis test or unpaired $t$-test. The level of statistical significance was set at $P<0.05$. 

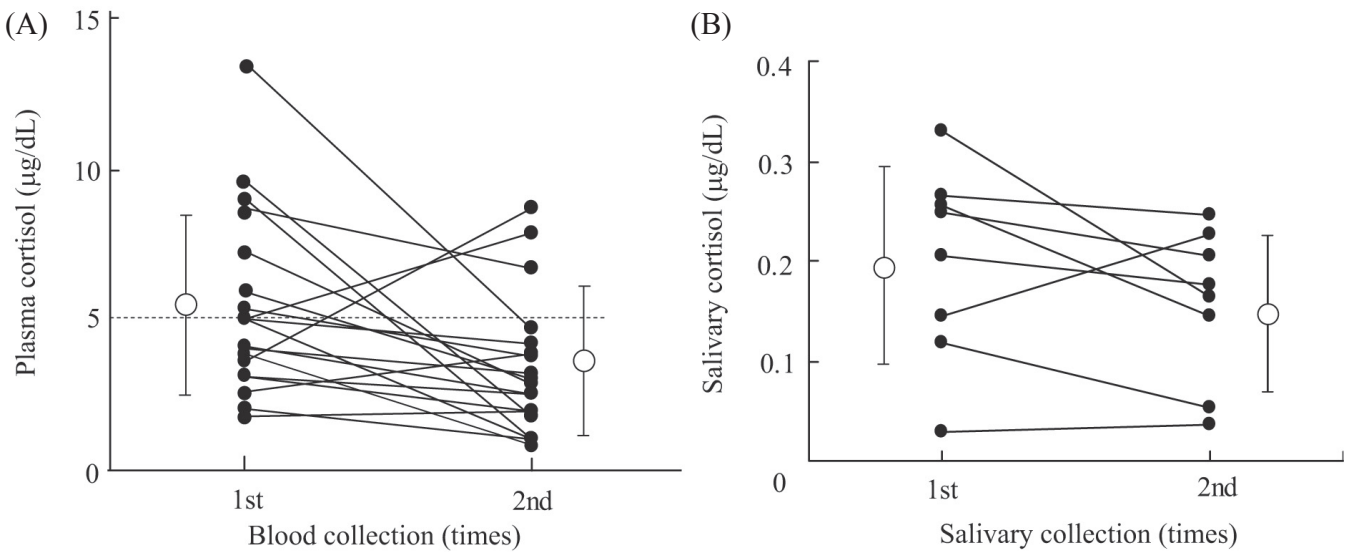

Fig. 1. Reproducibility of late-night plasma and salivary cortisol levels. Blood (A) and salivary (B) collection at 23:00 was performed twice: within three days of study entry (first time), and after one week (second time).

\section{Results}

\section{Reproducibility of plasma and salivary cortisol levels during night time sleep}

Plasma cortisol levels (mean $\pm \mathrm{SD}, 3.6 \pm 2.3 \mu \mathrm{g}$ / $\mathrm{dL}$ ) during night time sleep were significantly lower $(p<0.01)$ at the second measurement than at the first measurement $(5.7 \pm 3.1 \mu \mathrm{g} / \mathrm{dL})$ in 19 subjects with normal ACTH and cortisol secretory dynamics (Fig. 1A). There was no significant difference $(p=0.15)$ in salivary cortisol levels during night time sleep between at the first measurement (mean $\pm \mathrm{SD}, 0.20 \pm 0.10$ $\mu \mathrm{g} / \mathrm{dL})$ and at the second measurement $(0.16 \pm 0.08$ $\mu \mathrm{g} / \mathrm{dL}$ ) in 8 subjects (Fig. 1B).

\section{Correlation between plasma and salivary cortisol levels}

The Pearson's correlation coefficient (s) between plasma and salivary cortisol levels was 0.79 , which showed a significant correlation $(p<0.01)$ (Fig. 2A).

More than $0.4 \mu \mathrm{g} / \mathrm{dL}$ of salivary cortisol levels at night produced a sensitivity of $86 \%$ and a specificity of $100 \%$ in all Cushing's syndromes, including subclinical adrenal Cushing' syndrome and subclinical Cushing's disease (a sensitivity of 85\%) (Fig. 2B). When either plasma cortisol levels $(>5 \mu \mathrm{g} / \mathrm{dL})$ or salivary cortisol levels $(>0.4 \mu \mathrm{g} / \mathrm{dL})$ were considered as positive results, our study showed a sensitivity of $100 \%$ and a specificity of $82 \%$ in Cushing's syndrome (Fig. 2B).

Salivary cortisol ratio and urinary and plasma cortisol levels in Cushing's syndrome

Plasma cortisol levels at night were significantly higher in subclinical and overt Cushing's syndrome
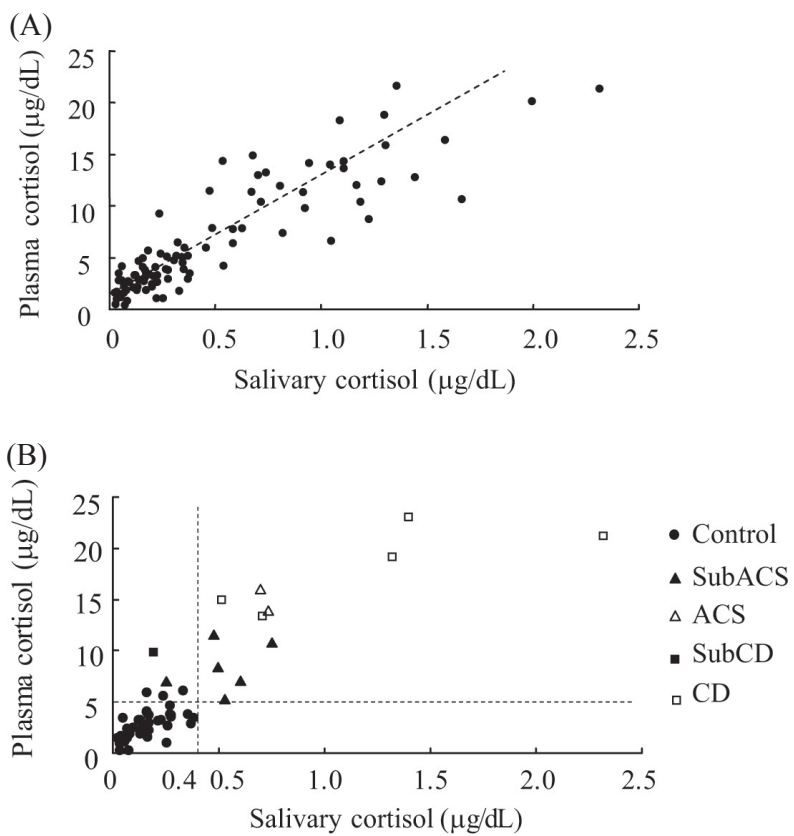

Fig. 2. Correlation between plasma and salivary cortisol levels. (A) Pearson's correlation analysis between plasma and salivary cortisol levels $(n=72)$. (B) Scatterplot of plasma and salivary cortisol levels in Cushing's syndrome. Closed circles, control $(\mathrm{n}=36)$; closed triangles, subclinical adrenal Cushing's syndrome $(\mathrm{n}=6)$; open triangles, adrenal Cushing's syndrome $(\mathrm{n}=2)$; closed squares, subclinical Cushing's disease $(\mathrm{n}=1)$; open squares, Cushing's disease $(n=5)$. A dotted line indicates our suggested cut-off level of either plasma or salivary cortisol.

than normal subjects (Fig. 3A). Plasma cortisol levels exceeded $5.0 \mu \mathrm{g} / \mathrm{dL}$ in 9 out of 10 cases of subclinical Cushing's syndrome, and in all cases of Cushing's syndrome (Fig. 3A). A plasma cortisol cut-off level of 

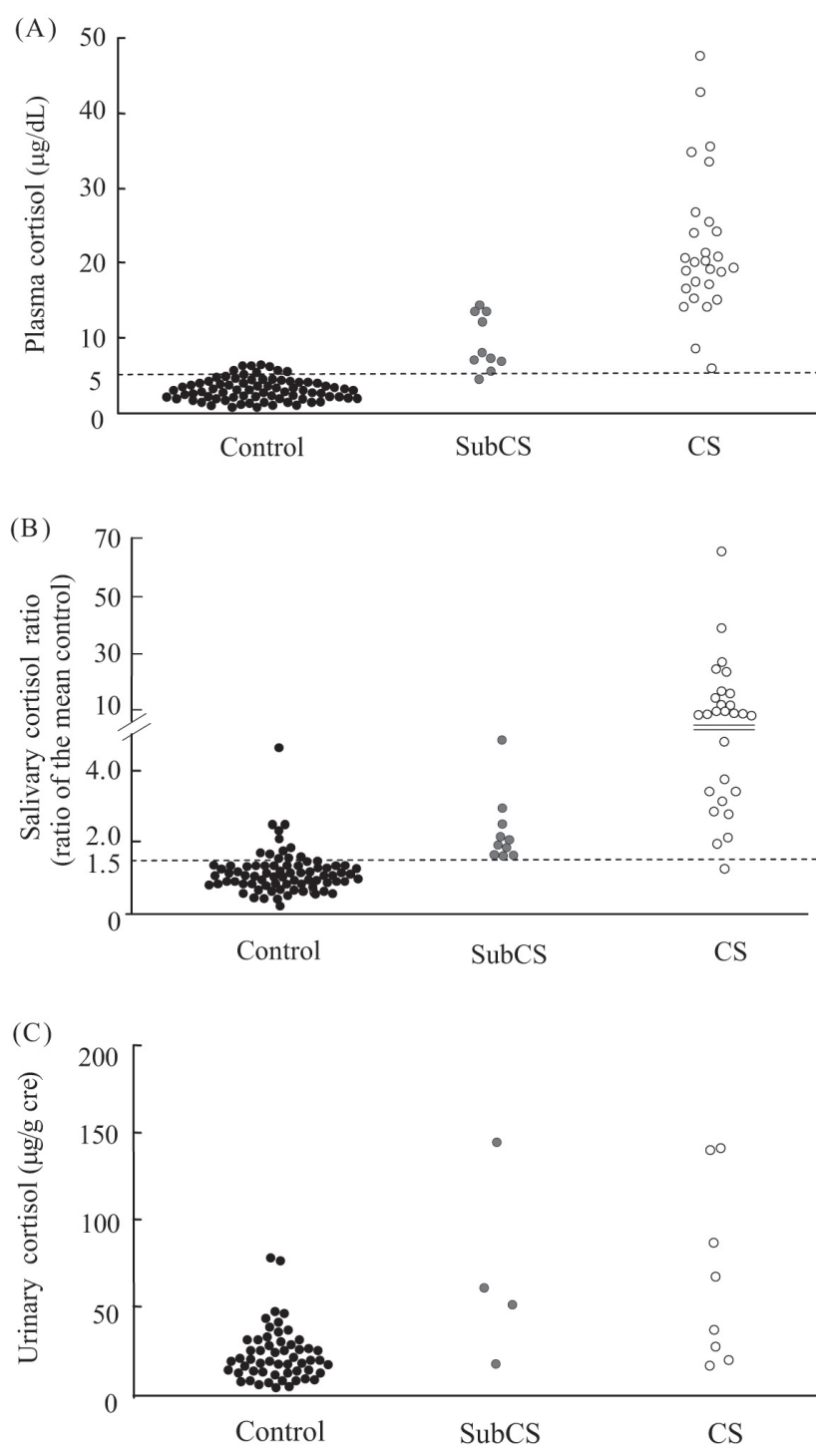

Fig. 3. Evaluation of cortisol levels in Cushing's syndrome. Twenty-seven subjects were diagnosed with Cushing's syndrome (CS), 10 with subclinical Cushing's syndrome, and 77 were used as control in the analysis of the salivary cortisol ratio. Blood, salivary, and then urinary samples were taken at 23:00. Among them, data on urinary cortisol levels were obtained from 67 subjects. (A) Plasma cortisol levels in subclinical Cushing's syndrome (SubCS) and Cushing's syndrome (CS). A dotted line indicates our suggested cut-off level of plasma cortisol. (B) Salivary cortisol ratio in subclinical Cushing's syndrome (SubCS) and Cushing's syndrome (CS). Salivary cortisol ratios are presented as a relative level, compared with the mean levels of healthy subjects in each institute. A dotted line indicates our suggested cutoff level of salivary cortisol ratio. (C) Urinary cortisol levels in subclinical Cushing's syndrome (SubCS) and Cushing's syndrome (CS).
$2.5 \mu \mathrm{g} / \mathrm{dL}$ showed a sensitivity of $100 \%$ and a specificity of $31 \%$ in all Cushing's syndrome cases, whereas a cut-off level of $5.0 \mu \mathrm{g} / \mathrm{dL}$ showed a sensitivity of $97 \%$ and a specificity of $88 \%$ in all Cushing's syndrome cases (Table 1).

In our study involving multiple institutes, salivary cortisol ratio was more than 1.5 in all subjects with subclinical Cushing's syndrome, and in 26 out of 27 cases of Cushing's syndrome (Fig. 3B). A ratio cutoff level of 1.5 showed a sensitivity of $97 \%$ (subclinical Cushing's syndrome, 96\%; subclinical Cushing's syndrome, $100 \%$ ) and a specificity of $88 \%$ in all Cushing's syndrome cases (Table 1).

Urinary cortisol levels at night showed overlapping results between Cushing's syndrome and controls (Fig. 3C). A urinary cortisol cut-off level of $18 \mu \mathrm{g} / \mathrm{g}$ cre showed a sensitivity of $100 \%$ and a specificity of $48 \%$ in all Cushing's syndrome cases (Table 1).

\section{Correlation between plasma cortisol levels and salivary cortisol ratios/urinary cortisol levels}

When all data from the four institutes were considered together (Table 2), there was no correlation between plasma and salivary cortisol levels $(r=0.23)$ (Fig. 4A). Next, we calculated salivary cortisol ratios as there appeared to be a correlation between plasma and salivary cortisol levels at each institute. The data showed a significant correlation between plasma cortisol levels and salivary cortisol ratios $(r=0.57)$ (Fig. 4B) but showed no significant correlation between plasma and urinary cortisol levels $(r=0.17)$ (Fig. 4C).

\section{Discussion}

Evaluation of cortisol level is important in the screening of Cushing's syndrome [16, 17]. New diagnostic criteria for Cushing's disease revised in 2007 found high cortisol levels ( $>5 \mu \mathrm{g} / \mathrm{dL})$ during night time sleeping to be indicative of ACTH-dependent Cushing's syndrome in Japan. However, the plasma cortisol sampling late at night is sometimes limited and stressful [13]. In this study, we evaluated the reproducibility of measuring plasma cortisol levels late at night. We found that plasma cortisol levels during night time sleep were significantly lower at the time of the second measurement than at the first time. This result suggests that high cortisol levels $(>5 \mu \mathrm{g} / \mathrm{dL}$ ) during night time sleep are indicative of Cushing's syndrome, although they may be artificially increased 
Table 1. Summary of the diagnostic tests in Cushing's syndrome (CS).

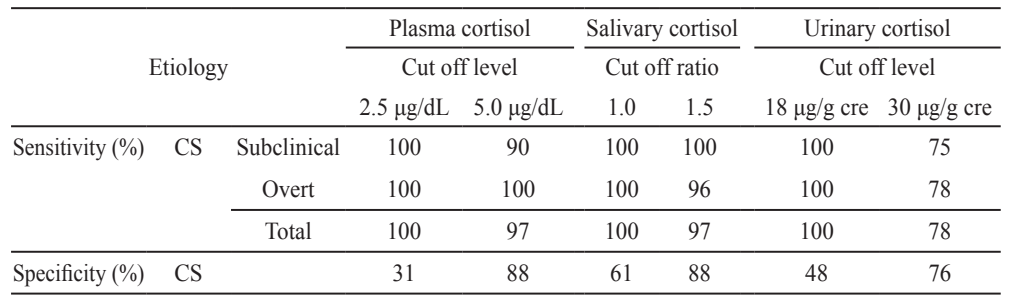

Table 2. Mean values of salivary cortisol in each institute.

\begin{tabular}{|c|c|c|c|c|}
\hline & \multicolumn{2}{|c|}{ Control } & \multicolumn{2}{|c|}{ Cushing's syndrome } \\
\hline & $\begin{array}{l}\text { Subjects } \\
(\mathrm{n}=77)\end{array}$ & $\begin{array}{c}\text { Mean } \pm \text { SD } \\
(\mu \mathrm{g} / \mathrm{dL})\end{array}$ & $\begin{array}{l}\text { Subjects } \\
(\mathrm{n}=37)\end{array}$ & $\begin{array}{c}\text { Mean } \pm \text { SD } \\
(\mu \mathrm{g} / \mathrm{dL})\end{array}$ \\
\hline Hirosaki & 25 & $0.21 \pm 0.10$ & 10 & $1.27 \pm 1.83$ \\
\hline Hamamatsu & 21 & $0.04 \pm 0.02$ & 14 & $0.92 \pm 1.53$ \\
\hline TokyoMD & 14 & $0.12 \pm 0.03$ & 13 & $1.31 \pm 2.11$ \\
\hline Kochi & 17 & $0.13 \pm 0.12$ & 0 & \\
\hline
\end{tabular}
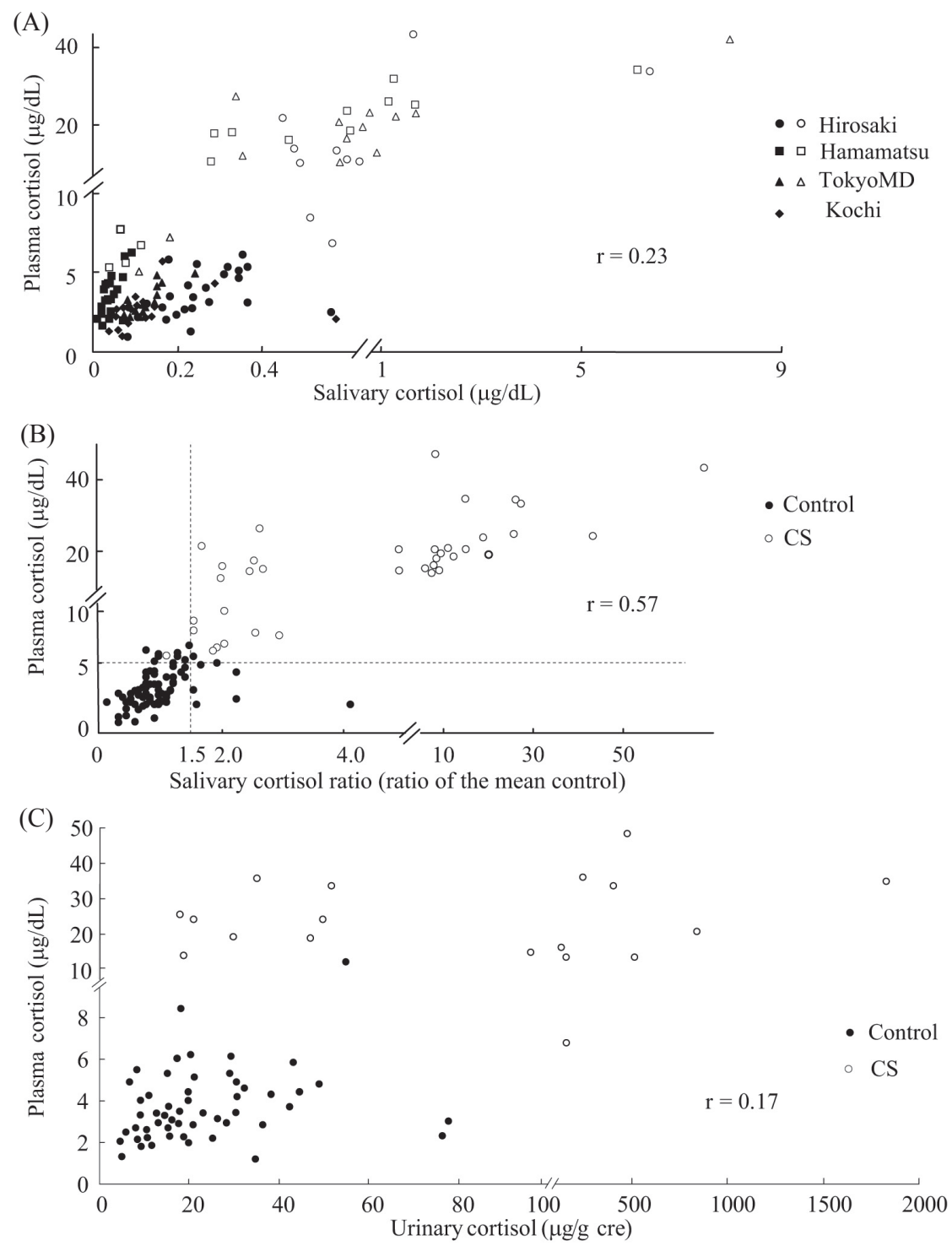

Fig. 4. Scatterplot of plasma and salivary cortisol levels. (A) Scatterplot of plasma and salivary cortisol levels in various institutions. Closed (control) and open (Cushing's syndrome, CS) circles, Hirosaki University Graduate School of Medicine; closed (control) and open (CS) squares, Hamamatsu University School of Medicine; closed (control) and open (CS) triangles, Tokyo Medical and Dental University Graduate School (TokyoMD); closed (control) lozenges, Kochi Medical School. (B) Scatterplot of plasma cortisol levels and salivary cortisol ratio in Cushing's syndrome (CS), including both subclinical and overt CS. Salivary cortisol ratios are presented as a relative level, compared with the mean levels of healthy subjects in each institute. A dotted line indicates our suggested cut-off level of either plasma cortisol or salivary cortisol ratio. (C) Scatterplot of plasma and urinary cortisol levels in Cushing's syndrome (CS), including both subclinical and overt CS. 
under stressful sampling condition.

The cut-off plasma cortisol level for overt Cushing's syndrome was found by our diagnostic criteria to be 5 $\mu \mathrm{g} / \mathrm{dL}$, while that for subclinical Cushing's syndrome was $2.5 \mu \mathrm{g} / \mathrm{dL}$. As the sensitivity of the $2.5 \mu \mathrm{g} / \mathrm{dL}$ cut-off level was $100 \%$ with a specificity of $31 \%$ in all Cushing's syndrome cases, while that of the $5.0 \mu \mathrm{g} / \mathrm{dL}$ cut-off level showed a sensitivity of $97 \%$ and a specificity of $88 \%$ in all Cushing's syndrome cases, this suggests that a cut-off level of $5.0 \mu \mathrm{g} / \mathrm{dL}$ is appropriate for diagnosis of all Cushing's syndrome cases.

Salivary cortisol sampling is simple and less invasive, so this method may be more useful and less stressful than blood collection $[12,13]$. In our study, late-night plasma and salivary cortisol levels showed a positive correlation. In fact, salivary cortisol concentrations are directly proportional to serum unbound cortisol concentration [18]. Our data, combined with a simple, stress-free, and non-invasive collection procedure, suggest that the salivary cortisol level is a more appropriate measure for the clinical assessment of adrenocortical function than the plasma cortisol level.

Salivary cortisol sampling is also found to be useful for the diagnosis of Cushing's syndrome as a late-night level of more than $0.4 \mu \mathrm{g} / \mathrm{dL}$ produced a sensitivity of $86 \%$ and a specificity of $100 \%$ in all Cushing's syndrome cases in our hospital. The method could also be important in the diagnosis of early or mild stage Cushing's syndrome, so-called subclinical Cushing's syndrome. Such diagnosis would contribute to the prevention of disease progression and accompanying complications. Inherent differences between assays cause difficulties in determining optimal diagnostic criteria; for example, the two different antibodybased techniques, enzyme-linked immunosorbent assay (ELISA) and RIA, previously gave different results from the same sample $[19,20]$.

Combining data from all four institutes in the current study found no correlation between plasma and salivary cortisol levels, because the basal values or normal ranges were quite different in each institute. However, we did observe a significant correlation between plasma cortisol levels and salivary cortisol ratios. Calculation of the ratio is particularly useful in the screening of Cushing's syndrome, since the cut-off ratio level of 1.5 showed a high sensitivity and specificity in both subclinical and overt Cushing's syndrome.

No significant correlation between plasma and urinary cortisol levels was observed in this study. The differences of cortisol clearance between blood and urine may cause the result. The cut-off level of $18 \mu \mathrm{g} /$ $\mathrm{g}$ cre in urinary cortisol showed a high sensitivity but low specificity in Cushing's syndrome, while the cutoff level of $30 \mu \mathrm{g} / \mathrm{g}$ cre showed a higher specificity and lower sensitivity. Taken together, late-night urinary cortisol levels offered weak information for the diagnosis of Cushing's syndrome.

In conclusion, high cortisol levels ( $>5 \mu \mathrm{g} / \mathrm{dL}$ ) during night time sleep are considered indicative of Cushing's syndrome, although night time plasma cortisol levels may be increased under stressful conditions. Salivary cortisol sampling is a less limited and stressful method for the evaluation of late-night free cortisol level. Salivary cortisol ratios are particularly useful for the screening of Cushing's syndrome because of high sensitivity and specificity in subclinical and overt Cushing's syndrome using a cut-off level of 1.5. The late-night salivary cortisol measurement is a primary method of choice in the screening of any patient suspected of having Cushing's syndrome.

\section{Acknowledgments}

This work was supported in part by Health and Labour Science Research Grants (Research on Measures for Intractable Diseases) from the Ministry of Health, Labour, and Welfare of Japan.

\section{References}

1. Newell-Price J, Besser M (2002) Evaluation of Cushing's syndrome. In: Blevins LS (ed) Cushing's syndrome. Kluwer academic publishers, Massachusetts: 71-97.

2. Findling JW, Raff H (2006) Cushing's Syndrome: Important issues in diagnosis and management. J Clin
Endocrinol Metab 91: 3746-3753.

3. Suda T (2002) Adrenal preclinical Cushing's syndrome. JMAJ 45: 172-174.

4. Chihara K (2007) Guidance of diagnosis and treatment in Cushing's disease. Reports 2006 on diencephalohypophyseal damage in Health and Labour Science 
Research: 130-132 (In Japanese).

5. Makras P, Toloumis G, Papadogias D, Kaltsas GA, Besser M (2006) The diagnosis and differential diagnosis of endogenous Cushing's syndrome. Hormones (Athens) 5: 231-250.

6. Oki Y, Hashimoto K, Hirata Y, Iwasaki Y, Nigawara T, Doi M, Sakihara S, Kageyama K, Suda T (2009) Development and Validation of a $0.5 \mathrm{mg}$ Dexamethasone Suppression Test as an Initial Screening Test for the Diagnosis of ACTH-dependent Cushing's Syndrome. Endocr J 56: 897-904.

7. Sakai Y, Horiba N, Tozawa F, Sakai K, Kuwayama A, Demura H, Suda T (1997) Desmopressin stimulation test for diagnosis of ACTH-dependent Cushing's syndrome. Endocr J 44: 687-695.

8. Malerbi DA, Mendonça BB, Liberman B, Toledo SP, Corradini MC, Cunha-Neto MB, Fragoso MC, Wajchenberg BL (1993) The desmopressin stimulation test in the differential diagnosis of Cushing's syndrome. Clin Endocrinol (Oxf) 38: 463-472.

9. Newell-Price J, Trainer P, Besser M, Grossman A (1998) The diagnosis and differential diagnosis of Cushing's syndrome and pseudo-Cushing's states. Endocr Rev 19: 647-672.

10. Makras P, Toloumis G, Papadogias D, Kaltsas GA, Besser M (2006) The diagnosis and differential diagnosis of endogenous Cushing's syndrome. Hormones (Athens) 5: 231-250.

11. Törnhage CJ (2009) Salivary cortisol for assessment of hypothalamic-pituitary-adrenal axis function. Neuroimmunomodulation 16: 284-289.

12. Evans PJ, Peters JR, Dyas J, Walker RF, Riad-Fahmy D, Hall R (1984) Salivary cortisol levels in true and apparent hypercortisolism. Clin Endocrinol (Oxf) 20:
$709-715$

13. Raff H (2009) Utility of salivary cortisol measurements in Cushing's syndrome and adrenal insufficiency. $J$ Clin Endocrinol Metab 94: 3647-3655.

14. Nieman LK, Biller BM, Findling JW, Newell-Price J, Savage MO, Stewart PM, Montori VM (2008) The diagnosis of Cushing's syndrome: an Endocrine Society Clinical Practice Guideline. J Clin Endocrinol Metab 93: $1526-1540$.

15. Suda T, Kageyama K, Nigawara T, Sakihara S (2009) Evaluation of diagnostic tests for ACTH-dependent Cushing's syndrome. Endocr J 56: 469-476.

16. Newell-Price J, Trainer P, Perry L, Wass J, Grossman A, Besser M (1995) A single sleeping midnight cortisol has $100 \%$ sensitivity for the diagnosis of Cushing's syndrome. Clin Endocrinol (Oxf) 43: 545-550.

17. Papanicolaou DA, Yanovski JA, Cutler GB Jr, Chrousos GP, Nieman LK (1998) A single midnight serum cortisol measurement distinguishes Cushing's syndrome from pseudo-Cushing states. J Clin Endocrinol Metab 83: 1163-1167.

18. Vining RF, McGinley RA, Maksvytis JJ, Ho KY (1983) Salivary cortisol: a better measure of adrenal cortical function than serum cortisol. Ann Clin Biochem 20: 329-335.

19. Raff H, Homar PJ, Burns EA (2002) Comparison of two methods for measuring salivary cortisol. Clin Chem 48: 207-208.

20. Baid SK, Sinaii N, Wade M, Rubino D, Nieman LK (2007) Radioimmunoassay and tandem mass spectrometry measurement of bedtime salivary cortisol levels: a comparison of assays to establish hypercortisolism. $J$ Clin Endocrinol Metab 92: 3102-3107. 